\title{
LA ATENCIÓN A LA DIVERSIDAD DESDE LA MUSICOTERAPIA
}

\author{
Adela Zahonero Rovira ${ }^{1}$
}

\section{RESUMEN}

Este trabajo es una invitación a la reflexión y profundización acerca del potencial que la Musicoterapia desempeña en el contexto Educativo cuando es utilizada como herramienta específica de apoyo a la Atención a la Diversidad de necesidades educativas de amplio espectro que se nos presentan en las Aulas. Partimos, para este planteamiento, de la experiencia práctica derivada de la aplicación de las posibilidades terapéuticas de la Música en Educación Secundaria que se viene llevando cabo, experimentalmente y de modo más o menos continuado, desde el curso 2003-2004 en ciertos Institutos de la Comunidad de Madrid, siendo su desarrollo objeto de alguna investigación ${ }^{2}$.

Partiendo de una metodología analítico- práctica se pone de manifiesto cómo la Musicoterapia incide favorablemente en las relaciones grupales, siendo un medio idóneo para atención de necesidades educativas y personales diversas de los miembros integrantes de los grupos receptores, que inciden en una mejora substancial del Clima relacional y de Convivencia del Aula, desde el que pueden transferirse aportes hacia la promoción de un ambiente más positivo en contextos más amplios, del Clima del propio Centro.

Palabras-clave: Diversidad, Musicoterapia, Clima de Convivencia, Clima de Aula.

\section{INTRODUCCIÓN}

En Educación Secundaria, se asiste, como en otros ámbitos sociales, a la presencia en las aulas de alumnado con intereses, motivaciones y capacidades muy distintas, entre los que se hallan personas procedentes de otras etnias y culturas, así como otras con problemas emocionales y/o de aprendizaje o bien con retraso en el desarrollo psicofísico y/o emocional, consecuencia de discapacidades de distinta etiología.

La Atención a la Diversidad supondría, pues, partir de esas distintas necesidades como indicadores de los apoyos que tal variedad de individuos precisan para atenderlas, brindando de esta forma a cada alumno el tipo y grado de ayuda real que precisa en aras de un adecuado progreso personal y académico.

\footnotetext{
1 Profesora de la Universidad de Alcalá. Departamento de Didáctica, Área de Didáctica y Organización Escolar. Guadalajara, (España). adela.zahonero@uah.es

2 Zahonero Rovira, Adela (2006) Influencia de la Musicoterapia en el Clima de Convivencia de los Institutos de Educación Secundaria. Tesis Doctoral. Universidad de Alcalá.
} 
Siguiendo la línea propuesta por Echeita ${ }^{3}$, al subrayar la importancia que las relaciones de apoyo mutuo entre los centros escolares y sus comunidades suponen para la educación inclusiva y la Atención a la Diversidad, y hasta qué punto ese conjugar los esfuerzos de padres, profesores, directores, alumnos, administradores y la comunidad local en un empeño común debe ser sostenido en el tiempo si se pretende recoger los anhelados frutos. Ahora bien, cuando se emprenden proyectos de innovación como el que presentamos, es evidente que la tarea requiere, indudablemente, de apoyos,...y no sólo de un espíritu creativo e innovador de los Equipos docentes. Ese apoyo representa tiempo y autonomía real para que los equipos educativos puedan tomar decisiones que se ajusten a las propias condiciones de los centros y su profesorado, y también comprensión por parte de las familias, los implicados y la Administración, de que los procesos de cambio también precisan de un espacio temporal, crean confusión, y no se pueden imponer desde fuera, sino facilitarlos para que afloren desde dentro de los propios equipos docentes (Fullan, 1991). Y, directamente vinculado a lo que nuestra propuesta de Atención a la Diversidad pretende, también significa apoyo la valoración real, de facto y no propagandística, de las áreas currículares que, como es el caso de la Música, de un modo explícito se ocupan del desarrollo de la sensibilidad artística... Difíciles tareas las de los apoyos en todas sus facetas, pero no imposibles con la adecuada voluntad...

En pocos años, la sociedad española ha asistido a un vertiginoso incremento de la presencia de inmigrantes, que llegan a nuestro país con la esperanza de un modo de vida mejor. Vienen, en la mayor parte de los casos, con hijos en edad escolar que suelen recibir su derecho a la educación en centros públicos. Muchas veces comienzan su actividad escolar cuando el año escolar ya ha dado comienzo, factor que añade dificultades aún más importantes a una integración social y lingüística compleja, sin obviar los problemas de aprendizaje asociados a las diferencias de currículo, niveles, y sistemas de enseñanza con respecto a sus países de origen.

Por ello, estos alumnos son más proclives a necesitar apoyos especiales que les ayuden a sentirse mejor, más adaptados a su entorno socioeducativo en primera instancia, y a la sociedad que les acoge después.

Además, debemos tener presente que, los cambios sociales también afectan a la profunda revisión de valores que está experimentando nuestra sociedad, lo que genera multitud de inestabilidades personales, muchas veces originadas dentro de las propias familias de los alumnos, especialmente sensibles en el momento de la adolescencia en

3 Echeita Sarrionandia, G. (2001)Conferencia "El proceso hacia la inclusión en educación" Universidad Autónoma de Madrid. 
el que se hallan. Es evidente que, cuando nos encontramos ante familias desestructuradas, se producen problemas en los adolescentes, tanto a nivel personal como en su capacidad de aprendizaje, y esas dificultades son proclives a su traslación en la presencia de conductas poco deseables dentro de las clases.

Por ello resulta apasionante el recurso, en el campo educativo, de las terapias artísticas, en este caso, a través de la Música, debido a las posibilidades comunicacionales y de expresión de la vida interior del individuo que brinda, haciendo que se considere como una opción en el tratamiento de personas en las que la comunicación verbal no es posible por limitaciones funcionales o psíquicas, o en aquéllas en que es importante la comunicación extraverbal.

\section{La Musicoterapia en Educación}

La Musicoterapia es el uso dosificado e intencional de la Música para promover cambios en la conducta dirigidos a una mejora del bienestar personal, Alvin (1987). Como profesión vinculada al campo de la salud, está, además, relacionada con la psicología, la educación, la educación especial, la psicoterapia y la medicina. Desde esta perspectiva, resulta sumamente complejo dar una descripción concreta y breve que englobe a todos los grupos de pacientes y los posibles métodos que se ven involucrados en la práctica musicoterapéutica moderna. Esta disciplina abarca a un mismo tiempo lo físico y lo emocional, las necesidades cognitivas y sociales de individuos de todas las edades, mejorando la calidad de vida de personas que están bien, y yendo al encuentro de las necesidades de niños y adultos con enfermedades o discapacidades. Las intervenciones musicoterapéuticas pueden dirigirse a la mejora del bienestar personal, al control de la ansiedad, al alivio de situaciones dolorosas, a la expresión de sentimientos, a la estimulación y/o conservación de memoria, la facilitación de la comunicación y la promoción de la rehabilitación física.

La investigación en Musicoterapia se apoya en una amplia variedad de propuestas vinculadas al campo de la educación y la salud. Cuando la enfocamos desde una óptica musical, los musicoterapeutas hacen uso de una metodología tal como escribir canciones, escuchar distintos tipos de Música, hacer Música, improvisar formando parte de un grupo comunitario, cantando, bailando, etc. En la mayor parte de los casos es muy difícil separar el hacer Música de la propia intervención terapéutica. La Música debe ser enfocada desde su perspectiva social, la de hacer algo junto a los demás, y ese es su significado más importante para nosotros, el de crecer uno mismo dentro de una interacción con otros, hacer algo que deja una reminiscencia... 
Ducourneau (1990) ${ }^{4}$ señala que, “educar a un niño es darle los medios para vivir, es decir, educarle para que pueda resolver los problemas que se le presenten”. La Musicoterapia utiliza la Música vinculándola a la capacidad de escucha, y desde esta perspectiva, puede ser un medio del que dotar al niño/alumno/ adolescente/ con una herramienta que le permita acceder con mayor facilidad a los conocimientos que sus profesores intentan transmitirle, si su capacidad para interpretar lo que le llega desde el mundo exterior a través de su oído está, por una u otra razón, disminuida. Dupré Nathan (1998:274) ${ }^{5}$ señala que "el oído es el intermediario entre el mundo objetivo de las vibraciones, y el subjetivo de las imágenes sonoras". Por tanto, la audición es un medio de conocimiento afectivo del exterior. No sólo es necesario que el oído esté bien desde la perspectiva fisiológico-anatómica, sino que debe ser correctamente utilizado.

El entrenamiento musical cultiva la atención y la memoria, lo que nos permite fijar un primer objetivo terapéutico-pedagógico: sensibilizar al alumno hacia el mundo de los sonidos, enseñándole a escuchar bien y a colocarse en posición de escucha. La persona sensibilizada al fenómeno sonoro, escucha el mundo de otra manera y se da cuenta de que todo alrededor de ella es variación, lo que se traducirá en una agudeza auditiva general diferente, y una actitud distinta hacia la lectura o la poesía. Pero además, a través de la Música podemos preparar de modo más eficaz a nuestros alumnos de cara a realizar las demás adquisiciones. Su estrecha relación con el lenguaje es evidente cuando se trata de la percepción y de los cuatro puntos centrales en los que ésta se centra: El timbre, la Altura, la Articulación, y la Energía articulatoria. Si nos ubicamos en una perspectiva más terapéuticomusical, sin embargo, nuestro objetivo prioritario, y complementario del anterior, sería ofrecer a los alumnos/ pacientes una intervención orientada hacia su desarrollo personal, emocional y comunicativo, al tiempo que crear un espacio idóneo que posibilite experiencias de tipo estético en un encuadre musical donde la Música actúa como vehículo facilitador de la expresión de sus emociones.

Por otra parte, tal y como nos dice Rogers "La Música permite la expresión de emociones en un contexto no amenazador y no verbal, facilitando la exploración destacando actitudes en un entorno seguro". Dados los problemas a los que debemos hacer frente mediante el lenguaje verbal, la Música permite un camino exclusivo para trabajar con la

4 En su obra Musicoterapia: La comunicación musical. Su función y métodos en terapia y reeducación. Edit. Edaf.Madrid

5 Dupré, Jean Paul (1998)L'Ėnclopédie vivante Nathan. Edit.Catherine Jardin. Paris

6 Rogers,C (1993) Research in Music Therapy with sexually abused clients, in PYNE, H (Edit.), One River, Many Currentes, Jessika Kingsley Publishers, p86-97. 
resistencia del paciente, con su negación o su baja autoestima. Esto, tiene repercusión directa sobre sus emociones, inicialmente a través de su proyección mediante los instrumentos: la interacción puede ser desarrollada mediante las palabras y la Música como emociones exteriorizadas a través de la improvisación, que pueden, entonces, ser atraídas hacia la consciencia del adolescente. De esa forma se pueden aprovechar los vínculos entre comportamiento y sentimientos y ser explorados.

Para concluir este primer apartado, vamos a sintetizar las razones por las que la Música puede convertirse en un instrumento terapéutico de Atención a la Diversidad en el contexto educativo de la siguiente forma, tal y como recogemos en nuestro trabajo, Zahonero, A.(2006:232):

1. La Música capta y mantiene la atención, estimulando y utilizando muchas zonas del cerebro de forma simultánea.

2. La Música nos ofrece un contexto divertido y lleno de significado para la repetición.

3. La música es fácil de adaptar a las habilidades de cada persona, reflejándolas.

4. La Música estructura el tiempo en el sentido de que podemos entenderla, haciéndonos que éste pase más deprisa.

5. La Música nos provee de un contexto social en el que sentirnos a salvo, estructurando el espacio en el que se establece la comunicación verbal, y sobre todo la no verbal.

6. La Música es una ayuda efectiva a la Memoria.

7. La Música apoya y estimula al movimiento.

8. La Música evoca recuerdos y emociones.

9. La Música, así como el silencio que conlleva, provee de una "retroalimentación no verbal inmediata".

10. La Música es una forma de permitir, que personas de todos los niveles de capacidad puedan participar de forma exitosa en las actividades hacia las que se les oriente.

La naturaleza no verbal de la Música la convierte en un medio de comunicación universal. El estímulo sonoro tiene un potencial único para penetrar en el cuerpo y la mente de las personas, sean cual sean sus condiciones o niveles intelectuales individuales, porque estimula los sentidos, evoca sentimientos, emociona, facilita respuestas fisiológicas y mentales, y suministra energía al cuerpo y la mente. Sus cualidades además presentan la facultad de organizar al individuo y al grupo, posibilitando al tiempo el aprendizaje y la adquisición de habilidades.

\section{2.- La Atención a la Diversidad desde la Musicoterapia}




\subsection{Musicoterapia y adolescencia}

La primera aproximación europea al ámbito de la Musicoterapia en educación viene de la mano de la británica Juliette Alvin (1987), si bien su enfoque, está fundamentalmente dirigido al contexto de la educación especial. En Francia, nos resulta especialmente interesante y útil para nuestra investigación, la visión psicohumanística y fenomenológica de la Musicoterapia como medio de apertura de los canales de información/comunicación en contextos educativos, que debemos al Profesor Ducourneau, quien dedica parte de sus estudios a la educación general, y lo mismo podríamos decir de Thayer (1989), en Estados Unidos, por lo que a la sistematización de problemas conductuales en alumnos no diagnosticados como "de educación especial", se refiere.

Son pocos los musicoterapeutas que, en la actualidad, dirigen sus esfuerzos a los adolescentes en contextos educativos y a lo que nosotros hemos denominado la atención de las necesidades específicas que en este ámbito se presentan., llegando casi a lo anecdótico si cabe el de aquéllos quienes se plantean la aplicación de las diferentes posibilidades de la Música para ayudar a que el adolescente encuentre su propia identidad. Sin embargo hay que destacar las experiencias pioneras de introducción de apoyos musicoterapéuticos que, patrocinados por la Universidad finlandesa de Turku y el profesor Jukko Tervo están llevándose a cabo en escuelas y Liceos de Finlandia, o las del Instituto Nordorff Robbins de Londres, junto a la de la Dra. Round de la Universidad de Anglia en el Reino Unido. Por ello, nos parecen tan interesantes las que comenzaron a llevarse a cabo en la Comunidad de Madrid, auque lo hicieran de una forma tan poco instucionalizada y balbuceante, y las que se mantienen sean tan aisladas.

Nuestra aportación, como terapeutas en un contexto educativo, es intentar mejorar la calidad de vida de estos adolescentes, teniendo siempre presente el momento tan peculiar en el que se encuentran, y las circunstancias añadidas que pueden contribuir a que esa fase de su desarrollo personal sea aún más compleja. En aras de esa calidad, nuestros objetivos habrán de enfocarse hacia la conjunción de tres componentes: Actividad, relaciones, y autoconfianza. La Música ayuda a promover las tres cosas, proporcionando una identidad flexible, al mismo tiempo que la consciencia de los sentimientos, la experiencia y expresión, que además tiene repercusión sobre la forma en que uno se abre a la otra gente, una de las mayores dificultades que tienen los adolescentes.

La forma de intervención musicoterapéutica más adecuada, en consonancia con los rasgos característicos de esta población, es la terapia grupal, sin exclusión de tareas y técnicas 
individuales cuando sea preciso, debido a la gran toma de conciencia de pertenecer a un grupo, y a la importancia que la socialización en esta etapa del desarrollo representa.

La música es un instrumento particularmente adecuado para la interacción social. Ofrece un amplio margen de actividades, desde un programa pasivo donde se escucha música hasta la participación activa en la danza y en la interpretación musical con diferentes instrumentos y atendiendo a diferentes estilos musicales, a través de las cuales el musicoterapeuta trabaja aspectos y desafíos a los que se enfrentan los adolescentes como:

- ¿Cómo puedo comprender quién soy ahora y quién seré en el futuro?

- ¿Cómo puedo alimentar y desarrollar relaciones positivas?

- ¿Cómo puedo desarrollar técnicas que me ayuden ante los desafíos, problemas, decisiones y elecciones del día a día?

- ¿Cómo puedo convertirme en un ser humano ético, activo, comprometido y y consecuente?

A través de la Musicoterapia se ofrece al adolescente una "Experiencia estructurada", " una Experiencia en la auto-organización” y una 'Experiencia en la relación con los demás “:

1. Experiencia estructurada:

La Música requiere una conducta cronológica, es un Arte en el tiempo.

- Objetiva de modo inmediato y continuo.

- Adaptada a la realidad.

- La música permite una conducta adecuada a la capacidad.

- Permite adaptar la conducta a las pautas de respuesta física.

- Permite adaptar la conducta a las pautas de respuesta psicológica.

- La música provoca una conducta orientada por lo afectivo.

- La música provoca una conducta elaborada de acuerdo a los sentidos.

- Requiere que se aumente el empleo de los sentidos

- Puede suscitar ideas y asociaciones extra-musicales.

2. Experiencia en la auto-organización:

- La Música posibilita la auto-expresión.

- La Música hace posible que las personas con discapacidad desarrollen conductas compensatorias. 
- La Música ofrece oportunidades de recibir recompensas y castigos socialmente aceptables.

- La Música hace posible elevar la autoestima.

- Hace posible tener experiencias de éxito.

- Hace sentirse necesario para otros.

- Eleva la estima de los demás.

3. Experiencia en la relación con los demás:

- La Música ofrece los medios para expresarse de un modo socialmente aceptable.

- Brinda al individuo la oportunidad de elegir su respuesta en los grupos.

- Proporciona oportunidades de asumir responsabilidades para con uno mismo y para con los demás.

- Aumenta la interacción social y la comunicación verbal y no verbal.

- Permite la cooperación y la competición en formas socialmente aceptables.

- Proporciona el entretenimiento y la recreación que requiere el ambiente terapéutico en general.

La capacidad energizante de la Música es una forma de activar un rango de sentimientos y elecciones, así como el modo de expresarlo. A veces, en su afán por "escapar" de la realidad, los adolescentes reprimen sus sentimientos, intentando no comunicarse, de lo que puede resultar una ruptura de sus relaciones con los demás y el entorno. Hacer Música juntos puede aportar un sentido de la aceptación, que permite al adolescente que se sienta escuchado, y entendido dentro de una estructura organizada desde la perspectiva musical. ${ }^{7}$ Además, la Musicoterapia en grupo permite al individuo aprender el valor de su música y la de los demás, al tmepo que se siente escuchado. El impacto que produce el sonido se percibe como un todo, y la forma en que cada uno lo hace es importante también para los demás miembros del grupo, porque se ven envueltos en una consciencia de sentimientos que los conduce a sus propias experiencias. Esto puede ayudarlos a diferenciar entre distintos estados emocionales, y a vincular emociones, comportamiento, y pensamiento.

Aunque se trabaje individualmente por razón de las características del tratamiento de un paciente, para el adolescente es importante y necesario compartir sus vivencias con los 
demás adolescentes, con el fin de apartar la idea de que sus problemas "sólo les pasan a ellos". De este modo, los procesos de cambio y desarrollo se producen mediante el reto y la escucha a los otros, aprendiendo a entenderse y aceptarse entre sí.

Al mismo tiempo, la Música es una forma de permitir el control al adolescente, de que sea autodisciplinado, y no permita la influencia de los demás. El terapeuta utiliza las técnicas de improvisación sobre dos dimensiones: por una parte la conciencia de uno mismo y los demás, y por otra, mediante la independencia de la expresión permitiendo al paciente una creatividad autónoma mientras se siente seguro. La experiencia de ser dinámico junto a otros, da al paciente sentimientos de libertad, posibilitándole la expresión de otros más fuertes. La experiencia musical actúa además como contenedor y transformador, puesto que la improvisación permite emerger a la auténtica persona, dentro del marco de una situación real vivida y compartida, que permite a los adolescentes ser conscientes de sus sentimientos y autopercibirse más vitales, a través del significado y el valor que el terapeuta da a sus experiencias. La Musicoterapia ayuda a incrementar la capacidad de tomar iniciativas, elegir, confiar, cuando se decide qué instrumento tocar, cómo integrarse en el grupo, lo que, en definitiva, eleva la autoestima, porque se toma el control de las situaciones y se mantiene la actividad. La participación, bien sea desde la escucha como tocando instrumentos, contribuye al desarrollo emocional, motórico, social, y del ego.

\subsection{La Atención a la Diversidad desde la Musicoterapia con un ejemplo práctico}

La experiencia que ilustra desde una perspectiva práctica este trabajo, se desarrolló a lo largo de un curso escolar, el 2004-2005 en un Instituto de Educación Secundaria de la Comunidad de Madrid. Contaba con el precedente de aplicación de Musicoterapia en el Centro el curso anterior, en el que habían participado como receptores también algunos profesores. El segundo curso se decidió optimizar la disponibilidad del recurso de una única musicoterapeuta, por lo que hubo un solo grupo de receptores, concretamente de necesidades específicas, en el que la Musicoterapia fue introducida como Adaptación Curricular de la asignatura de Música dentro de una agrupación ya de por sí de carácter experimental de PreDiversificación curricular de $2^{\circ}$ de Enseñanza Secundaria Obligatoria.

Las personas integrantes de este grupo eran catorce alumnos, ocho chicos y seis chicas, con una amplio abanico de necesidades educativas y personales, que abarcaban desde la integración sociolingüística, los problemas de absentismo derivados de familias 
desestructuradas, desatención, circunstancias sanitarias, desadaptación escolar, dificultades de aprendizaje y desfases curriculares marcados, problemas emocionales a, por supuesto, conductuales, que se reflejaban en comportamientos altamente disruptivos en el entorno escolar. Muchos de estos alumnos tenían problemas de relación en contexto familiar, las interrelaciones con sus iguales, o su autoconcepto. En algún caso concreto, su situación era especialmente vulnerable, próxima a la pre-delincuencia más o menos soterrada, y expuesta a otros comportamientos negativos e incluso autolesivos.

\subsubsection{Desarrollo de la actividad}

En su totalidad, los integrantes del grupo experimental objeto de nuestro estudio fueron propuestos por el Departamento de Orientación, y en muchos casos, tuvieron diagnóstico previo a causa de sus problemas de aprendizaje y / o actitudinales, contando por tanto con la aprobación de sus familias y del Servicio de Inspección para integrarse en el grupo específico. Excepto en especiales, la modalidad musicoterapéutica utilizada fue la grupal, o, en circunstancias puntuales, de dos personas simultáneamente. Las familias de los alumnos del grupo específico, así como los propios alumnos, fueron debidamente informadas de las características particulares de esta Adaptación.

El grupo recibió Musicoterapia durante todo el curso durante dos sesiones semanales. Se trataba de una agrupación realmente numerosa desde la perspectiva terapéutica, si bien fue la única posible desde la coyuntura experimental organizativa del Centro, y hubo de ser aceptada.

\subsubsection{Metodología}

La atención a la diversidad desde la Musicoterapia, conlleva la experimentación con el universo sonoro haciendo Música, escuchando las propias producciones $\mathrm{y}$, aprendiendo todo un abanico de habilidades sociales y personales que encuentran una vía privilegiada de trabajo a través de la experiencia de comunicación mediante un lenguaje no verbal, con distintas reglas y muchas menos restricciones que aquél. Consecuentemente, la metodología de desarrollo de las clases/sesiones se ha planteado desde la experiencia personal y grupal en torno a los fenómenos sonoros, con algunas referencias al lenguaje musical en el que se apoya la grafía musical convencional, pero sin perder en ningún momento el centro de gravedad de la experiencia vivida, personal, y activa, esto es, "hacer Música". 
De un modo más concreto, la Metodología de trabajo se desarrolló básicamente a través de la modalidad de participación activa dentro de un grupo, si bien, en algunos casos, tal y como se ha apuntado, se utilizó terapia individual.

La Musicoterapia se basa en la improvisación y expresión musical y/o sonora, en función de propuestas musicales por parte del musicoterapeuta, de carácter libre en algunos momentos, o a cargo de los propios integrantes del grupo. En otros casos, las actividades eran más estructuradas, pero siempre focalizadas a las interacciones que se producían entre los distintos miembros.

La planificación de actividades tenía como eje principal de su diseño los objetivos a corto, medio, y largo plazo, siempre condicionada a la situación del "aquí y ahora" de los alumnos en cada sesión, de forma que la intervención musicoterapéutica se adaptaba en cada momento a las necesidades del grupo o el alumno concreto. El grupo manifiesta "su momento" en cuanto a nivel de ansiedad, cansancio, atención, capacidad de interrelación y modo en que esta se expresa, y las actividades previamente diseñadas se amoldan a lo que el musicoterapeuta percibe de éste.

Es evidente que la observación es más compleja cuando se trata de intentar captar individualizadamente cada uno de los integrantes del grupo, y que las estrategias que se adoptan en las sesiones, intentan involucrar a la mayor parte de estas personas que no siempre coinciden en sus situaciones personales. En todas las sesiones se intenta alcanzar el "status" predominante del grupo, permitiendo la expresión individual dentro de éste,...y respetar, en la medida de lo posible, coyunturas particulares y puntuales de cada uno de los alumnos.

\subsubsection{Actividades.}

Las actividades se apoyaron totalmente en las vivencias personales en torno al sonido y la Música, y estuvieron basadas en la improvisación, convirtiendo a los participantes del grupo en artífices de su propia Música y ambiente sonoro sin que para ello, fuera preciso de ningún conocimiento musical ni instrumental previo. De hecho, este es el caso de los alumnos del grupo del estudio. Se trataba de tocar instrumentos de pequeña percusión, Orff y membranófonos, (o cualquier otro que estuviera a nuestro alcance), como medio de interacción con los demás a través de los sonidos que producía. En algún caso las actividades, dependiendo de la dinámica y proceso particular del grupo, demandaban una escucha más pasiva, de una Musicoterapia"receptiva", aunque siempre éste, como todos los grupos de terapia, estaba en constante evolución y los cambios observados abrían nuevas perspectivas 
hacia las que dirigir la actividad grupal. El tocar instrumentos juntos se aborda en Musicoterapia desde distintas perspectivas, que abarcan la improvisación libre, la dirigida dentro de una determinada estructura; la improvisación que siguen determinadas consignas para ambientar sonoramente diversas propuestas; e interpretaciones estructuradas en las que se requiere la atención y un resultado mínimo para valorar la incidencia que las acciones individuales tienen en el grupo, etc.

En algunos momentos, se introducía el trabajo con Música de la que los alumnos escuchan habitualmente y que ellos aportaban a las sesiones a su libre albedrío, que podía servir como base para la reflexión en otros aspectos, bien sea desde la perspectiva del significado del texto o del análisis de los parámetros musicales que se pudieran analizar.

También fue frecuente la realización de viajes sonoros, relajaciones y estimulaciones a través de la audición musical más o menos pasiva. Las primeras tenían lugar fundamentalmente tras las actividades de movimiento, o cuando, por distintas razones, parecían estar agitados o nerviosos, vinculándose entonces la metodología a técnicas de imágenes guiadas.

El movimiento es una actividad cotidiana en Musicoterapia, en este caso en su faceta más ligada a la libre autoexpresión, utilizándose trabajos rítmicos y de coordinación, con Música grabada y también en vivo, en ocasiones interpretada por un pequeño grupo de los propios pacientes para acompañar a sus compañeros, y en otros por la musicoterapeuta en el piano, la guitarra o con algún instrumento de percusión. En función del estado global de los miembros del grupo, también introducían elementos de expresión corporal, libre o con alguna coreografía simple, propuesta por la terapeuta o por alguno de los adolescentes.

También figuraron propuestas vinculadas a la Arteterapia, con recursos tales como dibujar, pintar o escribir lo que surgiera cuando se escuchaba una determinada Música, o bien mientras otra parte del grupo estaba improvisando. En determinadas ocasiones los alumnos reflejaron por escrito observaciones sobre el comportamiento musical de alguno de sus compañeros, adoptando así distintas perspectivas en su experiencia.

\subsubsection{Evaluación de la Experiencia musicoterapéutica}

Esta experiencia y su planificación hubieran quedado vacías y reducidas a una mera declaración de buenas intenciones, si no hubiera estado acompañada por el compromiso de seguimiento y valoración continuada y minuciosa de los procesos que tuvieron lugar a lo largo de su desarrollo. 
La evaluación es un elemento imprescindible tanto para la práctica educativa como para la musicoterapéutica, aún más valioso cuando es utilizado como instrumento de reflexión sobre los posibles cambios que se están produciendo, las estrategias utilizadas que han resultado eficaces, las que no, y por qué, y de un modo particular, para establecer el grado de cumplimiento de los objetivos trazados.

Concretamente, se utilizaron los siguientes instrumentos de Evaluación:

- Informe /Evaluación elaborado por la musicoterapeuta tras cada sesión, práctica habitual en Musicoterapia. En este documento se recoge lo más significativo de cada uno de los encuentros musicoterapéuticos, desde las personas que han participado, a una breve descripción de cómo lo han hecho, las interacciones que se han producido en el grupo, los instrumentos y cómo han sido utilizados, y el papel que la Música y el sonido representan dentro de ese contexto terapéutico. Así, se va dejando una constancia documental de sucesos que tienen lugar en un contexto en el que se intenta que exista una escasa presencia de lo verbal, lo que hace más importante aún este tipo de seguimiento.

- Supervisión externa: Todo terapeuta debe estar sujeto a que, de vez en cuando, su trabajo sea supervisado por otros profesionales que aportan su visión experta y/ o más objetiva, de las situaciones que se reflejan en el curso de las sesiones. Los musicoterapeutas trabajamos dentro de un grupo interdisciplinar, y esta supervisión, en el caso concreto del Proyecto que se desarrolló en el Instituto, suponía el refrendo de las actividades que se estaban llevando a cabo mediante el juicio y las observaciones de dos acreditadas y expertas musicoterapeutas, profesoras de la Universidad Autónoma de Madrid.

- Coordinación con la Tutora del grupo, y la Orientadora responsable de la selección de los alumnos, a fin de ir estableciendo sus propias evaluaciones escritas más globales de todos los procesos que tuvieron lugar entre los componentes del grupo.

- Grabaciones de las sesiones: Desde Diciembre de 2004, la mayor parte de las sesiones fueron grabadas, ya que el mejor modo de seguimiento era volver una y otra vez sobre lo que ocurría en cada una de las sesiones, y este registro audiovisual servía, lógicamente, para evaluar otros aspectos. Catorce personas son demasiadas para la observación, por parte de un solo terapeuta, de los procesos individuales de cada una de ellas, al mismo tiempo que los acontecimientos que se producen en el grupo. Sin coterapeuta, un grupo idóneo no debiera exceder de cinco pacientes. Por ello, resulta vital el apoyo audiovisual que brinda el poder registrar las sesiones en cámara de vídeo a fin de establecer valoraciones fiables y obrar en consecuencia. La utilización de las cintas de vídeo se justifica sólo en este contexto, si bien se considera un instrumento de evaluación de gran valor. 
- Cumplimentación de distintos cuestionarios a fin de curso, por parte de todos los implicados en la participación en la experiencia, en el que se recogían sus puntos de vista y su propia valoración de la misma. Esto implica a alumnos, Equipo de profesores del grupo, Jefatura de Estudios y Equipo Directivo, Departamento de Orientación, y Tutora. En ese sentido, pretendíamos que todos los profesores del grupo de alumnos participantes evaluaran también cuál había sido la incidencia, si es que la había habido, de la participación de sus alumnos en el Proyecto.

- Dado que los objetivos generales se esbozaban en torno a las interrelaciones humanas que se producían en el Instituto, y uno de los barómetros que mide de modo objetivo y externo los problemas de Convivencia que se plantean en estos centros educativos es el número de partes de amonestación que se les impone a los alumnos, se efectuó un seguimiento de la incidencia que esta medida estaba teniendo entre los alumnos de este grupo desde que el curso dio comienzo.

- Entrevistas personales realizadas entre final de Mayo y Julio con cada alumno, la tutora, el Equipo Docente, Orientadora, Jefatura de Estudios.

Finalmente, se elaboró un informe final con las conclusiones de todas las Evaluaciones parciales, conjuntamente por parte del Equipos Directivos, Docente y de Orientación, así como la Coordinadora del Proyecto en el Instituto, que fue incluido en la Memoria del Centro.

\section{Conclusiones:}

Supone un enorme esfuerzo, en un entorno en el que la tradición en educación musical en los Centros educativos generales se abre paso con suma dificultad, que los responsables políticos consideren la Música como herramienta efectiva en una Atención terapéutica a la Diversidad. Y probablemente, tal y como se estructuran los apoyos a este tipo de necesidades “distintas" desde la administración educativa en España, si esa tradición estuviera afianzada, $y$, en general hubiera una valoración de la importancia de la educación de la sensibilidad artística y su potencial para ayudar en los aprendizajes sobre las demás áreas, esta reflexión nos conduciría, como ocurre en Estados Unidos, en los centros públicos de Finlandia por ejemplo, o en alguno privado del Reino Unido, a entender la figura de los musicoterapeutas en contextos educativos como miembros de los Departamentos de Apoyos Psicopedagógicos (de Orientación, en nuestro caso) de Pedagogía Terapéutica.

De hecho, en la Comunidad de Madrid sigue pareciendo una anécdota, una experiencia aislada que, sin embargo de forma constante y discreta continúa obteniendo innegables frutos 
positivos sobre los progresos personales, ayuda efectiva a la integración en el Aula, mejora de relaciones entre los miembros del grupo, aumento de la autoestima, incremento del autoconcepto y autoconfianza personales cuyas consecuencias, en muchos de los casos, han generado una actitud más positiva con la que encarar sus aprendizajes, y por tanto se han visto reflejadas en mejores rendimientos académicos de un buen número de alumnos. Aquí nos hemos referido a una experiencia concreta, pero se han producido otras, en otros tantos Institutos, en los que, amén de refrendarse los aspectos puestos de relieve previamente, también han participado profesores como receptores, poniéndose de manifiesto cómo este tipo de intervenciones incide positivamente en la disminución de los grados de ansiedad del profesorado, mejorando también su actitud en su trabajo, y el Clima en el Centro.

Durante aquel curso fueron esos catorce alumnos de un grupo específico los que pudieron sentirse más valorados, más comprendidos, y en definitiva capaces de conseguir mejores resultados en sus estudios..., escuchados, y percibidos desde nuestra perspectiva... Ellos valoraron la experiencia de una forma muy positiva y especial. Su entorno, circundante, (incluyendo equipo docente, equipo directivo, padres, etc), también. "Crecimos" juntos, vivimos lo que $\mathrm{Amir}^{8}$ denomina "momentos mágicos" o significativos en Musicoterapia. En todo caso, situaciones como estas, en su carácter de sutíles se escapan frecuentemente a las alambradas tejidas por la semántica y los límites impuestos por las palabras, entrañando un sentido que marca profundamente otros desarrollos posteriores, y se extiende a otras dimensiones, más allá de esos alumnos que en su momento precisan de "adaptaciones" o “diversificaciones" de un currículo, alcanzando también en sus ramificaciones a aquéllos que hemos tenido la oportunidad de asistir a la apasionante tarea de los progresos personales que han logrado con ello. Ójala las resonancias de estas experiencias encuentren oídos lo suficientemente receptivos como para aprovechar el enorme potencial integrador y enriquecedor que, para nuestra sociedad, tan Diversa, un Idioma universal y sin fronteras como el de la Música, nos ofrece en el mundo educativo.

\section{BIBLIOGRAFÍA}

- Ahonen-ErikÁinen, H. (1999) "Different Forms of Music Therapy and Working Styles of Music Therapists. A Qualitative Study". Nordic Journal of Music Therapy, 8(2), 156-167.

- Aigen, K (1993). "The Music therapist as a cualitative researcher". Music Therapy. 12,16-39.

8 AMIR, D (1996)“La Experiencia de la Musicoterapia : Momentos significativos” en Beginning Dialogues, Primer Simposium Internacional sobre Investigación Cualitativa en Musicoterapia, Berlín 1996 . Edit. By Lagenberg, Aigen, Fromer. Barcelona Publishers 1996. (p.65) 
- $\quad$ Aigen, K (1996) "Here we are in Music: One year with adolescent creative Musictherapy group in Nordorff Robbins" Music Therapy Monograph series $\mathrm{n}^{\circ} 2$.

- Aigen, K 1996) “ La identidad cultural del Investigador" en Beginning Dialogues,Primer Simposium Internacional sobre Investigación Cualitativa en Musicoterapia, Berlín 1996 . Edit. By Lagenberg, Aigen, Fromer. Barcelona Publishers 1996.( p. 166, 167).

- Aigen, K (1996) “El papel de los Valores en Musicoterapia” en Beginning Dialogues ,Primer Simposium Internacional sobre Investigación Cualitativa en Musicoterapia, Berlín 1996 . Edit. By Lagenberg, Aigen, Fromer. Barcelona Publishers 1996.( p 166, 167).

- Aldrigde,, D (1992) "Phisyologische Verändungen beim splielen improvisierter Musik-Einige Vorslage für Vorschungsarbeiten". Musiktherapeutisch Umschau,3,( p174-186)

- Alvin, J.(1983) Musicoterapia Buenos Aires. Edit. Paidós.

- Amir, D(1996)“ La Experiencia de Musicoterapia : Momentos significativos” en Beginning Dialogues, Primer Simposium Internacional sobre Investigación Cualitativa en Musicoterapia, Berlin 1996 . Edit. By Lagenberg, Aigen, Fromer. Barcelona Publishers 1996.

- Ansdell, G.\& Pavlicevic, M. ( 2001) Beginnig Researh in the Arts Therapies . London.Jessica Kingsley Puiblishers.

- Benenzon, R. (2000) Musicoterapia: de la teoría a la práctica Barcelona. Paidos.

- Benenzon, R; GAINZA, V. y Wagner, G (1997) Sonido, Comunicación y Terapia Salamanca .Edi. Amarú.

- Betés de Toro ( Com)(2000) Fundamentos de Musicoterapia . Madrid. Edit. Morata.

- Bruscia, K (2002 ) Definiendo Musicoterapia. Salamanca. Edit. Agruparte.

- Bunt, L. y Hoskyns,S. (2004) The Hand Book of Music Therapy. New York. Edit Brunner Routledge.

- Cerezo Ramirez(1999). Conductas agresivas en la edad escolar. Aproximación teórica y metodológica. Propuestas de intervención. Madrid. Ed Pirámide.

- Cassity, M.D y Cassity, J. (2006) Multimodal Psychiatric Music Therapy for Adults,Adolescents, and Children. London. Jessica Kingsley Publishers.

- Crowe, B.(2004). "A Complexity Science- Based Theory and Phylosophy for Music Therapy Practice and Research. Music Therapy Today". ( Online) Vol 3. May 2004.

- Davies, A. Y Richards, E. (2002) Music Therapy and Group work, London. Jessica Kingsley Publishers.

- Davis,W., Gfeller, K.E, y Thaut, M. (2000) Introducción a la Musicoterapia, teoría y práctica . Barcelona. Edit. Boileau.

- De Baker, J (1993) "Containment un Music Therapy", in Heal y Wigram, Music Therapy in Health and education, London Jessica Kingsley Publishers Ltd, p32-39.

- "Declaración de Salamanca de principios, política y práctica para las NEE", Cuadernos de Integración Educativa, núm. 3, Dirección de Educación Especial, México, 1994.

- Defensor del pueblo (2000) "Violencia escolar: El maltrato entre iguales en la Educación Secundaria Obligatoria".

- Del Campo, P. (2000). "Music Therapy in Spain" In Dileo-Maranto, C. Music Therapy:International Perspectives. Pennsylvania: Jeffrey Books.

- Derpins, J.P(2001) La Música y el cerebro . Barcelona.Edit. Gedisa. Ciencias cognitivas/ Música. ( $1^{\mathrm{a}}$ Ed. Española 1989).

- Ducourneau, G (1990) Musicoterapia: la comunicación musical. Su función y métodos en terapia y reeducación Madrid. Edit. Edaf.

- Erikson (1968) Identity.Youth and Crisis, London. Faber \& Faber,

- Echeita Sarrionandia, G. (2001) Conferencia "El proceso hacia la inclusión en educación", I Congreso de Discapacidad Cognitiva. Medellín, Colombia. Septiembre, 2001.

- García, L. et al., (1999) Conferencia "Educación para todos y satisfacción de las necesidades básicas del aprendizaje"'"Elementos básicos de las adecuaciones curriculares", Jointien, 1990. Dirección General de Investigación Educativa de la Subsecretaría de Educación Básica y Normal/sEP, Fondo Mixto de Cooperación Técnica y Científica México-España, México,.

- Giroux, H (2006) "Inclusión y diversidad, dos categorías prioritarias en la educación", separata de la revista de educación Sinéctica del ITESO, de la Universidad de MC MJasters, en Canadá.

- Grocke, D, y Wigram, T. (2007)Receptive Methods in Music TherapyLondon. Jessica Kingsley Publishers 
- Guash, M. (1996) Madrid. La acción educativa en el ámbito de la inadaptación social: Hacia un modelo de intervención en libertad. Edit.Estel Ema-Studis.

- Hargreaves,D.J. (1998) Música y desarrollo psicológico . Barcelona. Edit. Graó.

- Lecourt, É.(2005) Análisis de Grupo y Musicoterapia. Col. Arte y Proceso. Vitoria. Ed. Agruparte.

- Leivinson, C.A.(2006) Resonando....Ecos. matices y disonancias en la práctica musicoterapéutica. Buenos Aires. Edt. Nobuko.

- López Fernández Cao, M. y Martínez Díez, N. (2006) Arteterapia. Conocimiento interior a través de la expresión artística. Madrid. Tutor, Psicología.

- Marina, J.A,(2001) Inteligencia, ética y valores para la convivencia”( ponencia), Congreso de Convivencia, Vitoria-Gasteiz.

- Martín Bris, M. (2000) Clima de trabajo y organizaciones que aprenden Rev. Educar, n 27. Separata. Servicio de Publicaciones de la Universidad Autónoma de Barcelona.

- Martín Brís, M. (2002) El profesorado y el cambio educativo. Madrid. Edit. Centro de Innovación Educativa. Fundación hogar del empleado.

- Mateos, L. (2004) Actividades musicales para atender a la diversidad. Madrid .ICCE.

- Mateos, L. A. (2002) "El área de música en niños con necesidades educativas Especiales". En R. Aranda (Ed.). Educación Especial: Adaptaciones curriculares para niños con N. E. E. Madrid. Prentice Hall- Pearson Educación.

- Meyer, L.B (2001) Emoción y significado en la Música . Madrid. Edit. Alianza Editorial.

- Mercadal-Brotons, M. (2000) "Modelo Conductista". En M. Betés de Toro Com). Fundamentos de Musicoterapia Madrid.Edit Morata.

- Moreno, J.J (2004) Activa tu Música interior (Musicoterapia y psicodrama)Barcelona.Edit. Herder.

- Narejo Alcázar, N. y Salazar Rodríguez, M. (2002). "Vías para abordar los conflictos en el aula". Revista Electrónica Interuniversitaria de Formación del Profesorado, 5(4).

- Newham, P. (1999) Using Voice and Movement in Therapy London. Jessica Kingsley Publishers.

- Nordorff, P y Robbinns, C. (1977) Creative Music Therapy. New York.The John Day Company. (p103).

- Odell, H.(2004) "Music Therapy with adolescents within a multidisciplinary team".En: Music therapyMind and Body, papers read at ones day conference, British Society for Music Therapy. (p1-6.)

- Papalia, M.(1996). Escritos sobre Música, Musicoterapia y Educación. Buenos Aires, Argentina. Jaxco editores.

- Pavlicevic, M.( 2003) Groups in Music. London ltd.Jessica Kingsley.

- Rickson,D., Watkins,W.(2003). "Music Therapy to Promote Prosocial Behaviors in Agressive adolescent-boy. A Pilot Study". Journal of Music Therapy.

- Riley, S (1999) Contemporany Art Therapy with Adolescents . London and Philadelphia.Jessica Kingsley Publishers.

- $\quad$ Rogers, C.(1964) Towards the Science of the Person.Behaivorism and Phenomenology. The University of Chicago Press. T.W. Wann. (P 8-11)

- $\quad$ Rodrigo, M.S.(2000) Musicoterapia: Terapia de Música y sonido Madrid. Edit.Musicalis.

- Round, C (1998) “An exploration of the role and function of Musictherapy in a multidisciplinary service for adolescents". A dissertation submited in partial fulfilment of the requirement of Anglia Polytechnic University for the degree of Master of Arts. Ed. Anglia University Press. UK.

- Ruud, E (1987) "Music as Comunication: A perspective from semiotics and Comunication Theory". En Ruud ( ed) Norsk Muskforlag, Oslo ( brazilian wedition by Summus Edit.1990.

- Ruud,E (1997) Music and the Quality of Life. Nordic Journal of Musictherapy, Volume 6, $\mathrm{n}^{\circ} 2$.

- Sabbatella, P. (2001). "La Investigación en Musicoterapia: Ecos de una Identidad en Desarrollo". Revista Española de Musicoterapia, 4, 32-41.

- Santos Guerra, M.A. (1990) Hacer visible lo cotidiano. Teoria y práctica de la evaluación cualitativa de los centros escolares. Madrid: Akal.

- Swanwick, K (1991) Música, pensamiento y Educación. Madrid.Edit Morata.

- $\quad$ Sneck, D. y Berger, D. (2005) The Music Effect. London. Jessica Kingsley Publishers

- Tervo, J ( 2005) "Music Therapy With Adolescents". Voices, Vol n5.

- Taylor, D.B. (1997) Biomedical Foundations of Music as Therapy. St. Louis. Music Magna Baton. 
- Thayer, Gaston, E. y OTROS(1989) Tratado de Musicoterapia México.Edit.Paidós Psiquiatría, Psicopatología y Psicosomática.

- Tomatis, A (1963) L'Oreille et le langage . ParísEdit Le Seuil.

- Tomatis, A(1989) Les troubles scolaires París. Edit Fixot.

- Trianes, M.V., Morena, M.L, y Muñoz, A.(1999) Madrid. Relaciones sociales y prevención de la inadaptación social y escolar. Edit. Aljibe.

- Wigran,T.(2004) Improvisation: Methods and Techniques for Music Therapy Clinicians,Educators, and Students London. Jessica Kingsley

- Wigram,T (1993)" Music Therapy in Health and Education”. Journal of Music Therapy.May 1993.

- Willems, E. (1984) Las bases Psicológicas de la educación musical. Buenos Aires.Edit.Eudeba.

- Weissberg,, R. P. (2000). "Social and emotional learning: A framework for promoting mental health and reducing risk behavior in children and youth". Journal of School Health, 70, 179-185.

- Wilson, D. B. Gottfredson, D. C. y Najaka, S. S. (2001). "School-based prevention of problem behaviors: A meta-analysis". Journal of Quantitative Criminology; 17,247-272.

- Yalom, I.( 2005) The gift of therapy. London .Edit, Piatkus Books.

- Zahonero Rovira, A (2004) "Musicoterapia en el Contexto de la Arteterapia" Revista del IES Villarejo de Salvanés "Sacando Punta"Madrid.

- Zahonero Rovira, A(2005) "La práctica de la Musicoterapia en centros de Secundaria" Memoria del Seminario propuesto por el CRIF "Las Acacias". Madrid, cursos 2003-2005.

- Zahonero Rovira, A. (2006) La Influencia de la Musicoterapia en el Clima de Convivencia de los Institutos de Educación Secundaria. Tesis Doctoral. Universidad de Alcalá de Henares. 